\title{
Reduccionismo y enfoque de sistemas: dos enfoques complementarios
}

\section{Kayninkun achikyachiy intichikawan: iskay achikyachiy tinkuykuna}

\section{Patotagatsigenga aike kantagagantsipage: pite kengagantsi kantapacha ogenganepage}

Recepción: 25 mayo 2020 Corregido: 16 junio 2020 Aprobación: 10 septiembre

Gerardo Hernández Chávez

Nacionalidad: Mexicana / Universidad Politécnica de Tlaxcala Correo: gerardo.hernandez@uptlax.edu.mx / ORCID: https://orcid.org/oooo-0002-1050-5139

Yazmin Hernández Chávez

Nacionalidad: Mexicana / Universidad Politécnica de Tlaxcala Correo: yazmin.hernandez@uptlax.edu.mx / ORCID: https://orcid.org/oooo-0003-4708-4689

\section{Resumen}

En este artículo se hace un esfuerzo teórico para enfatizar la complementariedad del reduccionismo y el enfoque de sistemas. Pues bien, la tradición científica por mucho tiempo creyó que para comprender algo, la mejor forma era dividiéndolo, es decir, separar sus partes para determinar como funcionan y a partir de ello, obtener conclusiones de los hechos observados. Sin embargo, el enfoque de sistemas es una forma de entender las relaciones y el contexto que, por supuesto, está interesado en las partes, pero principalmente en términos de cómo dan lugar y mantienen en existencia una nueva entidad que es el todo. Por lo tanto, ambos enfoques son complementarios.

\section{Palabras clave:}

Reduccionismo, enfoque de sistemas y sistemas.

\section{Lisichiku limaykuna:}

Intichiyku, kaaninkuna achikyaachi kaaninkunawan.

\section{Nibarintsi katingaro:}

Patotagantsi, kengagantsi

aike Kengagantsi.

\section{Datos de los autores}

Gerardo Hernández Chávez es docente e investigador en enfoque de sistemas en general, sistemas de calidad y recursos humanos. Doctor en Administración Gerencial por el Instituto de Estudios Universitarios, Ciudad de Puebla, México. Maestro en Ciencias de la Calidad por la Universidad Autónoma de Tlaxcala, México.

Yazmin Hernández Chávez es docente e investigadora en enfoque de sistemas en general, sistemas de calidad y recursos humanos. Doctora en Administración Gerencial por el Instituto de Estudios Universitarios, Ciudad de Puebla, México. Maestra en Ciencias de la Calidad por la Universidad Autónoma de Tlaxcala, México. 


\section{Reductionism and the Systems Approach: Two Complementary Approaches}

\begin{abstract}
In this article it makes a theoretical effort to emphasize the complementarity of reductionism and the systems approach. Well, the scientific tradition for a long time believed that to understand something, the best way was to divide it, that is, to separate its parts to determine how they work and, from there, to draw conclusions from the observed facts. However, the systems approach is a way of understanding relationships and context, which of course is interested in the parts, but mainly in terms of how they bring about and maintain a new entity that is the whole, and therefore both approaches are complementary.
\end{abstract}

\section{Keywords}

reductionism, systems and the systems approach.

\section{Reducionismo e enfoque sistêmica: dois enfonques complementares}

\section{Resumo}

Neste artigo fazemos um esforço teórico para enfatizar a complementaridade do reducionismo e do enfoque sistêmica. Bem, a tradição científica por muito tempo acreditou que para entender algo a melhor maneira era dividindo-a, ou seja, separar suas partes para determinar como funcionam e a partir daí obter conclusões dos fatos observados. No entanto, a abordagem sistêmica é uma forma de entender relacionamentos e contextos que, claro, está interessado nas partes, mas principalmente em termos de como elas dão origem e mantêm na existência uma nova entidade que é o todo. Portanto, ambas as abordagens são complementares.

\section{Palavras-chave:} reducionismo, enfoque sistêmica e sistemas. 


\section{Introducción}

El hombre en el transcurso de su historia ha desarrollado dos enfoques aparentemente opuestos en la forma de entender una realidad. Por un lado, el paradigma de la ciencia clásica, explicaría un fenómeno en términos de elementos aislables. Por otra parte, la biología y las ciencias sociales Ilegaron a la conclusión de que éste no era satisfactorio. No obstante, el argumento de Aristóteles de que el todo es más que la suma de las partes, abriría el camino para comprender un todo organizado e interrelacionado.

Se revisará principalmente los conceptos de reduccionismo y enfoque de sistemas tratando de explicar que ambos enfoques son complementarios, además, se expone una nueva definición de enfoque de sistemas propuesta por Arnold y Wade (2015), que explica desde la perspectiva de sistemas cada uno de los elementos que comprende dicha definición.

La motivación para escribir este artículo radica en la importancia de destacar la complementariedad del enfoque reduccionista y el enfoque de sistemas. El artículo esta dividido en cuatro apartados; el primero, contiene los antecedentes de la perspectiva analítica; el segundo, explica qué es el reduccionismo y expone los tres tipos de reduccionismo; el tercero, esclarece el concepto del enfoque de sistemas; el cuarto, se expone por qué ambos enfoques son complementarios. Finalmente se presenta las conclusiones.

\section{Antecedentes}

Desde sus inicios el hombre enfrentó diversos obstáculos para sobrevivir y desarrollarse en sociedad. Por consiguiente, se dio cuenta que debía comprender la realidad en la que vivía, a fin de transformarla a través de cuestionamientos que le permitiera resolver los diversos problemas que enfrentaba. Como consecuencia de ello, desarrollo enfoques que le pudieran dar una explicación racional desde las partes hasta la totalidad. Ahora bien, la explicación de la realidad es compleja, debido a que uno de los rasgos prevalecientes del pensamiento moderno ha sido la tendencia analítica, que consiste en la descomposición progresiva del objeto hasta obtener sus unidades más simples, para comprender de esa manera el objeto de estudio.

Esta orientación analítica predominó en las principales corrientes filosóficas. Por ejemplo, a la física le servía como paradigma de conocimiento científico, pues, demostraba responder a relaciones causales directas entre un número reducido de entidades simples. Los fenómenos evidentemente más complejos lograban explicarse mediante su reducción analítica y descomposición en las partes que lo integraban. El análisis mostraba tal fuerza, que muchas veces se identificaba como si fuese sinónimo de conocer (Morín, 2001; Fisher, 2001). Por otra parte, para la biología resultaba cada vez más evidente que el esquema reduccionista impedía una adecuada explicación de los fenómenos de la vida. Los biólogos tendían progresivamente a aceptar la idea de que la clave para explicar la materia viva es el reconocimiento de su nivel de organización. Descubrían también que en la medida en que era desagregado en sus componentes químicos y físicos más simples, no era posible dar cuenta del fenómeno propiamente biológico, puesto que lo biológico no negaba la plena validez de la física o de la química, pero parecía constituirse en un nivel muy diferente, al interior de una jerarquía de niveles de complejidad. La emergencia de nuevos problemas en niveles superiores de complejidad resultaba ser un problema mayor para la ciencia, que el método analítico reduccionista no era capaz de resolver. La biología, por tanto, había comenzado a desarrollar modalidades de pensamiento capaces de estudiar el comportamiento de unidades complejas (Flood \& Jackson, 1991; Jac- 
kson, 2003). Es así que surge la necesidad y viabilidad de un enfoque de sistemas, pues una orientación mecanicista que hacía énfasis en las vías causales y aislables resultaba insuficiente para explicar fenómenos como la organización, regulación y otros procesos biológicos que tienen propiedades de los sistemas vivientes (Bertalanffy, 2004; Gigch, 2008).

Recapitulando, el reduccionismo que emergió del pensamiento analítico y el concepto de totalidad no son incompatibles entre si, considerando que para el estudio de los fenómenos tienen propósitos similares, es decir, son enfoques complementarios (Ackoff, 2008). Por esto, surgió la necesidad de obtener un enfoque que permitiera dar cuenta de la complejidad de ciertos fenómenos de la realidad observada, a la cual se nombro "enfoque sistémico", como una alternativa que, de acuerdo a Bertalanffy (2004), proporcionaría los principios que podrían ser adaptables a los sistemas de forma general sin importar su naturaleza, ya sea física, biológica o sociológica.

\section{¿Qué es el reduccionismo?}

El reduccionismo, de acuerdo a Viniegra (2014), es "una postura epistemológica que sostiene que el conocimiento de lo complejo debe ser, obligadamente, a través de sus componentes más simples, o que un sistema complejo solamente puede explicarse por la reducción hasta sus partes fundamentales" (p. 253). En otras palabras, describir las partes que conforman una máquina es el primer requisito para la comprensión de su funcionamiento; por ejemplo, si queremos describir el funcionamiento de un automóvil, estudiaremos las partes y mecanismos que permiten comprender el movimiento del auto. Es decir, "analizamos" qué significa fragmentar o descomponer el fenómeno u objeto que deseamos comprender, al cual, Descartes consideró como la primera premisa de su método de conocimiento; la segunda premisa, la nombró como "síntesis", asumiendo que consistía en integrar sus componentes para comprender la totalidad. Sin embargo, el análisis ganó aceptación con el tiempo y se convirtió en la única tradición del quehacer científico, excluyendo a la síntesis (Descartes, 2007).

El análisis es el primer momento del método de Descartes. Dada una dificultad, para plantear un problema era preciso ante todo considerarlo en bloque y dividirlo en tantas partes como fuera posible. Pero, surgían preguntas como: ¿En cuántas partes dividirlo? ¿Hasta dónde ha de llegar el fraccionamiento de la dificultad? ¿Dónde deberá detenerse la división?. La división deberá detenerse, argumenta Descartes, cuando hallemos la presencia de elementos del problema que puedan ser conocidos inmediatamente como verdaderos y de cuya verdad no puede caber duda alguna. Tales elementos simples son las ideas claras y distintas.

No obstante, señalan Cornejo y Viana (1998) y Viniegra (2014) que es importante distinguir los diferentes tipos del reduccionismo, que regularmente reconocen los autores en tres clases: la primera, es la ontológica, que consiste en la creencia de que la realidad está compuesta de un número mínimo de clases de entidades o sustancias; esto es, que los organismos están compuestos exclusivamente de partes no vivientes, pues Ayala (1984) expresa que ningún tipo de substancia o residuo de otro tipo queda después de que han tenido en cuenta todos los átomos que constituyen un organismo; la segunda, es la teórica, que se refiere a que los conceptos de un campo de investigación pueden y deben ser reducirlos a los de otro campo con un nivel de complejidad inferior; o lo que es lo mismo, que la conexión entre las teorías se logra a través de la demostración de que los principios de una teoría pueden ser explicados por los principios de otra teoría o rama de la ciencia que contiene mayor generalidad; la tercera, es la metodológica, que sostiene que la mejor estrategia científica es intentar reducir las explicaciones de los objetos a las entidades más pequeñas posibles, es decir, que 
la mejor estrategia de investigación, es estudiar los fenómenos vitales en niveles cada vez más bajos de complejidad, para llegar finalmente al nivel de los átomos y las moléculas. Ahora bien, los tres tipos de reduccionismo están presentes, de manera explicita e implícita, donde la verdad de la máquina viviente radica en lo infinitamente pequeño; así, de acuerdo al modelo mecánico-causal, para explicar un fenómeno es necesario descubrir los mecanismos causales que lo producen. Lo que significa que un mecanismo, se basa en las relaciones mecánicas de causa-efecto y desde esta visión analiza las situaciones, considerando la posibilidad de reducir los problemas a pocas variables, o a un determinado número de cadenas causales lineales.

En definitiva, la aportación del reduccionismo y el mecanicismo para las diferentes áreas de la ciencias, como la física, la química y las matemáticas, ha sido muy significativa, no así en las ciencias biológicas y sociales, donde dicho enfoque no ha considerado algunos fundamentos teleológicos, tales como la organización y la capacidad de dirección, considerados fundamentales para el análisis y comprensión de los fenómenos (Malagón y Prager, 2001). En definitiva, el reduccionismo es un proceso lógico de simplificación que está unido a la indagación de lo fundamental.

\section{El enfoque de sistemas}

Un enfoque, de acuerdo a Bunge y Ardila (2002), es "una manera de ver las cosas (por ejemplo, las personas) o las ideas (por ejemplo, las conjeturas) y, en consecuencia, también de tratar los problemas relativos a ellas" (p. 54). Es así que intentamos entender la realidad y que normalmente lo hacemos como se mencionó anteriormente, a través del enfoque reduccionista, que utiliza la división para explicar los fenómenos en un grado elemental, es decir, separarlos de tal modo que facilitará su estudio en un nivel de especialización, ya que ésta forma de abordar los fenómenos empíricos supone que es mejor tener un conocimiento específico y profundo de elementos más pequeños y mejor definidos, que un conocimiento general y abstracto de elementos más grandes e insuficientemente definidos; aunque el enfoque de sistemas tiende hacia la aplicación de una perspectiva holística, pues sostiene que no se resuelve un problema separándolo, sino que se debe considerar como parte de un sistema mayor, debido a que la suma de los criterios aplicados a las partes rara vez es igual a los criterios del todo. Por consiguiente, el enfoque de sistemas centra su atención en el todo, y en las complejas interrelaciones de sus partes constituyentes. Esta forma de ver no es una alternativa, sino un complemento a la forma especializada. Es más completo, e incorpora la perspectiva especializada como un aspecto de una concepción general (Laslo y Krippner, 1998).

Entre las acepciones que Gigch (2008) otorga al término enfoque de sistemas están las siguientes: una metodología de diseño, un marco conceptual común, un nuevo método científico, una teoría de la organización, un sistema de administración y la aplicación de la Teoría General de Sistemas. Por lo que, el enfoque de sistemas es apropiado para analizar y estructurar problemas complejos de sistemas sociales. Dado que éste consiste en un conjunto de subsistemas, planes y medidas de actuación para cumplir con un objetivo.

Como puede inferirse, entonces, el enfoque de sistemas es una forma de pensar en sistemas de todo tipo. No es una disciplina con fronteras definidas, lo cual, señala Korn (2019), es una de sus debilidades, pues comprende un marco conceptual interdisciplinario que puede adaptarse a diferentes áreas del conocimiento (Shaked y Schechter, 2017). La característica esencial de esta forma de pensar es que el pensamiento interviene desde el inicio para dictar la manera en que nosotros describimos lo que pensamos hacer. Es decir, debemos preguntarnos desde el inicio cómo 
pensar acerca de un sistema y nuestra forma de pensar nos dirá cómo describiremos el sistema (Islas y Lelis, 2007). Ahora bien, esta flexibilidad y versatilidad ventajosas para el enfoque sistémico ha llevado a una amplia gama de definiciones que a continuación se presenta en la tabla 1.

\section{Tabla 1}

Definiciones de enfoque de sistemas

\begin{tabular}{|c|c|}
\hline Autor & Definición \\
\hline $\begin{array}{c}\text { (O'Connor y } \\
\text { McDermott 1998, p. 18). }\end{array}$ & $\begin{array}{l}\text { Es un método de identificar algunas reglas, algunas series de patrones y } \\
\text { sucesos para prepararnos de cara al futuro e influir sobre él en alguna medida. } \\
\text { Nos aporta cierto control. }\end{array}$ \\
\hline (Senge 2010, p. 91 ) & $\begin{array}{l}\text { Una disciplina para ver totalidades. Es un marco para ver interrelaciones } \\
\text { en lugar de cosas, para ver patrones de cambio en lugar de "instantáneas" } \\
\text { estáticas. Es un conjunto de principios generales. También es un conjunto de } \\
\text { herramientas y técnicas específicas. }\end{array}$ \\
\hline (Stroh 2015, p. 16). & $\begin{array}{l}\text { Es la capacidad de comprender interconexiones para alcanzar un propósito } \\
\text { deseado. }\end{array}$ \\
\hline $\begin{array}{c}\text { (Arnold y Wade } 2015, \\
\text { p. } 675 \text { ). }\end{array}$ & $\begin{array}{l}\text { Un conjunto de habilidades analíticas sinérgicas utilizadas para mejorar } \\
\text { la capacidad de identificar y comprender los sistemas, predecir sus } \\
\text { comportamientos y diseñar modificaciones para producir los efectos } \\
\text { deseados. Estas habilidades trabajan juntas como un sistema. }\end{array}$ \\
\hline (Mobus, 2018, p. 13) & $\begin{array}{l}\text { Es la capacidad cognitiva de una persona para percibir la totalidad de un } \\
\text { sistema, para percibir las conexiones entre elementos y otros componentes } \\
\text { con los que interactúa causalmente, y para percibir la composición interna } \\
\text { de los subelementos, ellos mismos interconectados e interactuando para } \\
\text { producir el sistema. }\end{array}$ \\
\hline
\end{tabular}

Fuente: Elaboración propia con información de los autores.

Las definiciones de enfoque de sistemas comparten dos características comunes: por un lado, observa interrelaciones entre componentes y, por otro lado, que cada componente del sistema forma parte del todo. Por su parte, Arnold y Wade (2015) exponen una definición más amplia y actualizada del enfoque de sistemas, describiéndolo como "un conjunto de habilidades analíticas sinérgicas que se utilizan para mejorar la capacidad de identificar y comprender los sistemas, predecir sus comportamientos y diseñar modificaciones para producir los efectos deseados, donde dichas habilidades trabajan juntas como un sistema" (675). Los términos expresados se refieren a lo siguiente:

- Sistemas: grupos o combinaciones de elementos interrelacionados, interdependientes o interactivos que forman entidades colectivas.

- Sinérgico: es la interacción de elementos de una manera que, cuando se combinan, producen un efecto total que es mayor que la suma de los elementos individuales.

- Habilidades analíticas: habilidades que proporcionan la capacidad de visualizar, articular y resolver problemas y conceptos complejos y sin complicaciones, y tomar decisiones que sean sensatas y se basen en la información disponible.

- Comprender: estar completamente familiarizado con; aprehender claramente el carácter, la naturaleza o las sutilezas.

- Predecir: predecir como una consecuencia deducible.

- Diseñar modificaciones: para idear, planificar o elaborar cambios o ajustes.

Ésta definición del enfoque sistémico lo define como un sistema, sintetizando las competencias de dicho enfoque. 


\section{La complementariedad}

El enfoque de sistemas es un método para resolver problemas, donde estos no se pueden reducir a simples relaciones de causa y efecto, pues requiere una comprensión más amplia de las interacciones, de las relaciones y los comportamientos entre los elementos que caracterizan al sistema. Un sistema menciona Ackoff (2008) es un todo que no debe ser dividido en partes independientes; de ahí que, se derivan dos propiedades importantes de los sistemas. La primera, un sistema tiene propiedades que desaparecen cuando se separan del sistema; la segunda, cada sistema tiene propiedades esenciales, que no tiene ninguna de sus partes. Es así, que las propiedades esenciales de un sistema derivan de las interacciones de sus partes, por eso, cuando un sistema es separado pierde sus propiedades esenciales. Por lo tanto, un sistema no puede ser comprendido por medio del análisis o reduccionismo. A continuación se presenta el orden del enfoque analítico y de sistemas a tratar de entender un problema en tabla 2.

Tabla 2

Comparativo de enfoques

\begin{tabular}{ll}
\hline \multicolumn{1}{c}{ Enfoque analítico } & \multicolumn{1}{c}{ Enfoque de sistemas } \\
\hline - Descomposición de lo que va a ser explicado. & $\begin{array}{l}\text { - Identificar un todo que contenga un sistema. } \\
\text { - Explicación de la conducta o propiedades de las } \\
\text { partes, tomadas por separado. }\end{array}$ \\
$\begin{array}{l}\text { - Combinación de estas explicaciones en la conducta o las propiedades del todo } \\
\text { explicación del todo. }\end{array}$ & $\begin{array}{l}\text { que lo contiene. } \\
\text { Explicar la conducta o las propiedades del } \\
\text { objeto que va a ser explicado, en términos de } \\
\text { sus funciones dentro del todo. }\end{array}$ \\
\hline
\end{tabular}

Fuente: Ackoff (2008:30).

En el pensamiento analítico, el objeto que va a ser explicado es tratado como un todo que se va a descomponer. Por ejemplo, cuando tratamos de comprender un automóvil, regularmente empezamos de descomponerlo en términos de cuatro llantas, características del motor, equipo de sonido, tipo de luces, color y tamaño. En el enfoque de sistemas el objeto que se va a estudiar es considerado como parte de un todo contenedor y se debe comprender en términos de su función, es decir, preguntarnos para qué sirve, en este caso, el automóvil es un medio del transporte. Por consiguiente, el enfoque de sistemas es un enfoque totalizador que pone juntas las cosas y esta es la clave del enfoque de sistemas (Lara, 1990; Churchman, 1973).

Estos dos enfoques señala Ackoff (2008) no deberían producir resultados conflictivos o contradictorios, pues son complementarios; el análisis se enfoca en la estructura y revela cómo trabajan las cosas. La síntesis se centra en la función: descubre por qué operan las cosas y cómo lo hacen. Así, el análisis crea conocimiento, mientras que la síntesis origina comprensión. Con el primero se logra describir, mientras que el segundo permite explicar.

\section{Conclusión}

A manera de conclusión, cabe decir que en el pensamiento analítico, el objeto que va a ser explicado es tratado como un todo que se va a desmembrar, mientras en el enfoque sistémico el objeto que se va a estudiar es considerado como parte de un todo contenedor. El primero reduce el foco del investigador, mientras que el segundo lo amplía. Estos dos criterios, como se ha expuesto, son complementarios. El desarrollo de esta complementariedad es una de las tareas más importantes del pensamiento de sistemas; pues el análisis se enfoca sobre la estructura, es decir, revela cómo funcionan las cosas, y la síntesis se concentra en la función de descubrir cómo actúan las cosas. Así el análisis produce conocimiento, mientras que la síntesis genera comprensión. 
Referencias bibliográficas

Ackoff, R. (2008). Planificación de la empresa del futuro. Limusa.

Arnold, R.D. \& Wade, J.P. (2015). A definition of systems thinking: A systems approach, Procedia Computer Science. Elsevier Masson SAS, 44, pp. 669-678. https://doi.org/ 10.1016/j. procs.2015.03.050

Ayala, F.J. (1984). Relaciones ontológicas, metodológicas y epistemológicas entre biología y la física. Contextos, 11(3), 7-20.

Bertalanffy, L.V. (2004). Teoría general de los sistemas. Fondo de Cultura Económica.

Bunge, M. \& Ardila, R. (2002). Filosofía de la psicología. Siglo Veintiuno Editores.

Churchman, C.W. (1973). El enfoque de sistemas. Diana.

Cornejo, R. y de Viana, M.L. (1998). Reduccionismo: La vigencia de un antiguo problema. Epistemología e Historia de la Ciencia, Selección de trabajos de la VIII Jornadas, 4(4), 70-75.

Descartes, R. (2007). El discurso del método. Ediciones Akal.

Fischer, A. (2001). Critical thinking: An introduction. University of Cambridge.

Flood, R.L. \& Jackson, M.C. (1991). Creative problem solving: Total systems intervention. John Wiley \& Son.

Gigch, J. P. (2008). Teoría general de sistemas. Trillas.

Islas, V. \& Lelis, M. (2007). Análisis de los sistemas de transporte. SCT. https://www.imt.mx/ archivos/Publicaciones/PublicacionTecnica/pt307.pdf

Jackson, M.C. (2003). Systems thinking: Creative holism for manager. Wiley \& Son.

Korn, J. (2019). Crisis in systems thinking, Kybernetes, 49(7), 1915-1934. https://doi. org/10.1108/K-01-2019-0026

Lara, F. (1990). Metodología para la planeación de sistemas: Un enfoque prospectivo. UNAM.

Laszlo, A. \& Krippner, S. (1998). Systems theories: Their origins, foundations and development, Advances in Psychology, 26, 47-74. https://doi.org/10.1016/So166-4115(98)80017-4

Malagón, R. y Prager, M. (2001). El enfoque de sistemas: Una opción para el análisis de la unidades de producción agrícola. Feriva S.A.

Mobus, G.E. (2018). Teaching systems thinking to general education students, Ecological Modeling, 373(January), 13-21. https://doi.org/10.1016/j.ecolmodel.2018.01.013

Morín, E. (2001). Introducción al pensamiento complejo. Gedisa.

O'Connor, J. \& McDermott, I. (1998). Introducción al pensamiento sistémico: Recursos esenciales para la creatividad y la resolución de problemas. Urano.

Senge, P. (2010). La quinta disciplina: El arte y la práctica de la organización abierta al aprendizaje, 2 da Edición. Granica.

Shaked, H. \& Schechter, C. (2017). Definitions and development of systems thinking. Procedia Computer Science. Springer International Publishing AG. https://doi.org/ 10.1007/978-3-31953571-5_2

Stroh, D.H. (2015). Systems thinking for social change: A practical guide to solving complex problems, avoiding unintended consequences, and achieving lasting results. Chelsea Green Publishing.

Viniegra, L. (2014). El reduccionismo científico y el control de las conciencias. Parte I. Boletín Médico de Hospital Infantil de México, 71(4), 252-257. 